\title{
Valeurs bouchères des zébus à Madagascar
}

\author{
par J. GILIBERT (*)
}

\begin{abstract}
RESUME
L'auteur étudie comparativement les valeurs bouchères de zébus malgaches élevés au pâturage naturel, au pâturage naturel complémenté, en feed-lot, et en fosse. Il étudie ensuite dans les conditions de pâturage naturel complémenté ou non, puis en feed-lot des animaux métıs Zébu Brahman $\times$ Zébu Malgache. Un lot est constitué par des métis Zébu Malgache $\times$ Frison (ou Normand).

L'alimentation intensive et les croisements amènent une amélioration de la qualité des carcasses.
\end{abstract}

Madagascar exporte de la viande bovine depuis un siècle, viande essentiellement issue d'animaux Zébu autochtones. Les animaux ou bien sont élevés selon le mode extensif, ou bien subissent une embouche paysannale, avant leur abattage, décrite par SARNIGUET et collab. (13), selon des circuits traditionnels que l'on peut schématiser sur la carte tirée de LACROUTS et collab. (8).

L'ensemble de ces déplacements étant effectués à pied jusqu'à présent, on peut constater l'importance de ce facteur, les troupeaux parcourant couramment $500 \mathrm{~km}$.

Le développement d'industries de transformation sur les zones de productions agricoles importantes (riz, coton, arachides, sucre) permet de disposer de sous-produits en zone de naisseurs et près des lieux d'exportation. Elles vont permettre l'installation d'unités de finition industrielle de bovins; les avantages seront considérables puisque ces unités permettront d'éviter l'abattage d'un grand nombre d'animaux maigres, comme c'est le cas actuellement et d'ajouter à chaque carcasse traitée une plusvalue importante.

Quelques études ont été consacrées à la valeur bouchère du zébu malgache, notam-

(*) Ingénieur agronome I.N.A.

I.E.M.V.T., Région de recherches de Madagascar. ment par CHRETIEN (3) et à celle des zébus engraissés par METZGER et HAMON (10).

L'I.E.M.V.T. a mis en place un protocole de contrôle de carcasses depuis 1965 afin de préciser les normes de finition et permettre le départ de ces unités sur des bases saines. Les résultats à ce jour en sont donnés dans la présente publication qui comportera une étude des contenus digestifs, des rendements et de caractéristiques des carcasses.

L'amélioration génétique du cheptel bovin est un mode complémentaire des précédents pour l'augmentation de la production de viande. Les croisements à partir de races laitières, surtout Normande et Frisonne, sont pratiqués depuis longtemps sur les Hauts Plateaux. Le croisement avec la race Brahman du Texas est pratiqué depuis 1956 en différentes régions et dans les grands ranchs d'Etat créés ces dernières années; c'est un animal Zébu extrêmement rustique, dont la production, même en milieu défavorable, est un progrès considérable par rapport au bétail local.

Les conditions écologiques de l'Tle et les modes d'élevage et de commercialisation étant très variés, il convient de préciser, pour la bonne compréhension, l'amplitude de variation des facteurs qui seront étudiés : 





- L'âge d'abattage des bovins variera de 18 mois pour des métis engraissés intensivement à 12 ans pour des Zébus Malgaches originaires du Sud.

- Les poids vifs varieront de $311 \mathrm{~kg}$ pour des Zébus Malgaches insuffisants à $591 \mathrm{~kg}$ pour des métis en bon état.

- L'alimentation varie dans les proportions les plus larges en partant d'animaux d'extensif, dehors toute l'année sur de mauvais pâturages sans aucune supplémentation, avec une saison sèche pouvant durer jusqu'à huit mois, en passant par un mode d'élevage identique mais dans des conditions écologiques (climat, sol, topographie) plus favorables, par une embouche paysannale à base d'herbe et de manioc, par une finition où la proportion de concentré dans la ration est de plus en plus importante, cette finition pouvant être courte (quatre mois) ou longue (huit, douze mois) pour arriver à une technique de "taurillon précoce ").

On conçoit les variations considérables qui résulteront de ces différents facteurs sur les résultats de rendements et de carcasses.

Les animaux étudiés ici proviennent en forte majorité des deux Centres de Recherches de Kianjasoa et Miadana qu'il convient de situer sommairement (voir carte).

TABLEAU $\mathrm{N}^{\circ} \mathrm{I}$

\begin{tabular}{|c|c|c|c|c|c|}
\hline & Altitude & P1uviométrie & $\begin{array}{c}\text { Durée saison } \\
\text { sèche }\end{array}$ & Sols & $\begin{array}{c}\text { Distance du lieu } \\
\text { d'abattage }\end{array}$ \\
\hline Kianjasoa & $850 \mathrm{~m}$ & $1.600 \mathrm{~mm}$ & 5 mois $1 / 2$ & acides & $180 \mathrm{~km}$ \\
\hline Miadana & $40 \mathrm{~m}$ & $1.400 \mathrm{~mm}$ & 6 mois $1 / 2$ & sédimentaires & $520 \mathrm{~km}$ \\
\hline
\end{tabular}

L'étude ci-après permettra de déterminer la possibilité d'exporter de la viande soit sous forme de pans entiers pour lesquels il faut disposer de carcasses d'au moins $200 \mathrm{~kg}$, soit sous forme de désossé congelé pour lequel des carcasses de $160 \mathrm{~kg}$ peuvent suffire.

Pour la réalisation de ce travail nous avons utilisé les ouvrages de LACROUTS et collab. (8) et de SARNIGUET et collab. (13) en ce qui concerne la commercialisation du bétail; de SERRES et collab. (14) pour les questions d'engraissement à Madagascar; de CHAUDIEU et BONNEVILLE (2), DUMONT (5), DUMONT et collab. $(6,7)$, LE GUELTE $(9)$, CRAPLET (4), RENOU (11) pour la technologie des viandes.

\section{MATERIEL ET METHODES}

Dix-neuf lots d'animaux de boucherie ont été contrôlés à ce jour selon un protocole identique. Il s'agit des groupes d'animaux suivants : $\mathbf{Z H}_{1}$ : Zébus Malgaches d'herbe de 4 ans 9 mois, élevés à Kianjasoa, 11 animaux, 1969.

$\mathrm{ZH}_{2}$ : Zébus Malgaches d'herbe de 8 ans achetés au marché de Tsiroanomandidy, 4 animaux, 1966.
$\mathrm{ZC}_{1}$ : Zébus Malgaches de 19 mois finis au concentré rationné pendant 10 mois à Miadana, 10 animaux, 1970.

$\mathrm{ZC}_{2}$ : Zébus Malgaches de 3 ans 3 mois, finis au concentré rationné pendant 1 an à Kianjasoa, 6 animaux, 1969.

$\mathrm{ZC}_{3}$ : Zébus Malgaches de 4 ans finis pendant 6 mois au concentré rationné à Kianjasoa, 9 animaux, 1968.

$\mathrm{ZC}_{4}$ : Zébus Malgaches de 4 ans à l'herbe et manioc vert, Kianjasoa, 4 animaux, 1969.

$\mathrm{ZC}_{\bar{\jmath}}$ : Zébus Malgaches de 4 ans finis au concentré ad libitum pendant 6 mois à Kianjasoa, 10 animaux, 1967.

$Z_{6}$ : Zébus Malgaches de 7 ans 7 mois, finis au concentré rationné pendant 3 mois (insuffisant) à Kianjasoa, 8 animaux, 1968.

$\mathrm{ZC}_{\mathrm{i}}$ : Zébus Malgaches de 8 ans 6 mois finis au concentré rationné pendant 3 mois (insuffisant) à Kianjasoa, 10 animaux, 1968.

$\mathrm{ZC}_{8}$ : Zébus Malgaches de 9 ans 3 mois finis au concentré rationné pendant 4 mois à Kianjasoa, 8 animaux, 1969. 
$\mathrm{ZC}_{y}$ : Zébus Malgaches de 12 ans, dits Zébus de fosse, achetés aux environs de Tananarive, 2 animaux, 1968.

$\mathrm{MH}_{1}$ : Métis $1 / 2$ Brahman d'herbe de 3 ans 6 mois, élevés à Kianjasoa, 4 animaux, 1966.

$\mathrm{MH}_{2}$ : Métis 3/4 Brahman d'herbe de 3 ans 6 mois, élevés à Kianjasoa, 4 animaux, 1966.

$\mathrm{MH}_{3}$ : Métis $1 / 2$ Brahman d'herbe de 4 ans 6 mois, élevés à Miadana, 7 animaux, 1968.

$\mathrm{MH}_{4}$ : Métis 3/4 Brahman d'herbe de 4 ans 9 mois, élevés à Miadana, 9 animaux, 1969.

$\mathrm{MH}_{5}$ : Métis 1/2 Brahman d'herbe de 5 ans 6 mois, élevés à Kianjasoa, 8 animaux, 1968.

$\mathrm{MH}_{6}$ : Métis 3/4 Brahman d'herbe de 5 ans 6 mois, élevés à Kianjasoa, 9 animaux, 1968.

$\mathrm{MC}_{1}$ : Taurillons métis Frisons de 18 mois finis au concentré rationné pendant 8 mois, 9 animaux, Tananarive, 1969.

$\mathrm{MC}_{2}$ : Métis 5/8 Brahman de 22 mois finis au concentré rationné pendant 6 mois à Kianjasoa, 10 animaux, 1967.

Ces 19 lots représentant 142 animaux ont été abattus à l'abattoir de la S.E.V.I.M.A. à Tananarive et ont subi les contrôles suivants :

- Pesée au départ du lieu d'ólevage.

- Pesée avant abattage.

- Poids du tube digestif plein.

- Poids du tube digestif vide.

- Poids de la carcasse chaude (avec gras de rognon et queue).

- Poids de la carcasse froide.

- Longueur de la carcasse entre le bord antérieur de la symphyse pubienne et le bord antérieur du milieu de la première côte.

- Epaisseur de la cuisse à la sonde au niveau de la symphyse pubienne.

- Epaisseur du plat de côte entre la $7^{\mathrm{e}}$ et la $8^{\mathrm{e}}$ côte, au milieu de la longueur des côtes.

- Poids de l'arrière coupé à la $5^{\text {e }}$ côte.

- Poids de l'avant coupé à la $5^{\mathrm{e}}$ côte.

- Poids des deux gras de rognons.

- Poids de la bosse.

- Poids des deux pans traités.

- Observation visuelle du profil ischio tarsien, carcasse ressuyée pendue.
- Appréciation visuelle de la couverture de graisse.

- Appréciation de la couleur de la graisse.

- Désossage et parage du gras, industriellement, donnant le rendement en viande de la carcasse, pour la majorité des lots.

\section{CONTENUS DIGESTIFS ET RENDEMENTS}

Un des critères les plus importants, pour l'étude de la valeur bouchère consiste en J'évaluation du rendement en carcasse; immédiatement le technicien se heurte à une difficulté provenant du poids du contenu digestif qui est élevé et très variable chez les animaux polygastriques. Les rendements utilisés sont au nombre de trois pour cette raison:

- Le rendement commercial $=$ poids de carcasse froide divisé par poids vif à la ferme, multiplié par 100.

- Le rendement à jeun = poids de la carcasse froide divisé par poids vif à jeun, multiplié par 100.

- Le rendement “ vrai $"=$ poids de la carcasse froide divisé par poids vif moins lc poids des contenus digestifs, multiplié par 100.

- La définition du terme «à jeun » n'est pas très précise et varie d'un pays à l'autre selon les habitudes de commercialisation; on peut dire qu'en moyenne, il s'agit d'un jeûne hydrique de 24 heures.

D'autre part, des études sont fréquemment effectuées concernant les pertes de poids subies par les animaux entre le départ et l'abattage, ce problème étant surtout important dans les pays où les animaux effectuent une partie du trajet à pied. Or, l'analyse de ces résultats est difficile car il s'agit de faire la part du poids perdu par la carcasse, le seul ayant une incjdence économique réelle, et la part du poids perdu du fait de la vidange digestive. De plus, même si les animaux avaient subi une marche épuisante et un jeûne prolongé, le contenu digestif à l'arrivée ne serait pas nul.

Il a paru utile de ne pas dissocier l'étude du poids du contenu digestif de celle des rende- 
ments, seul le rendement vrai étant indépendant du contenu digestif.

\section{A. INFLUENCE \\ DE CERTAINS FACTEURS \\ SUR LE POIDS TOTAL \\ DE CONTENU DIGESTIF}

La totalité des animaux ayant voyagé en camion entre le lieu d'élevage et le lieu d'abat- tage, on admettra que la perte de poids des carcasses est nulle, bien que ce ne soit pas strictement vrai.

Le poids des contenus digestifs au départ sera alors mesuré par la perte de poids pendant le transport augmentée du poids des contenus digestifs restant dans l'animal abattu.

Les résultats utilisés pour l'étude des contenus digestifs sont donnés dans le tableau II.

TABLEAU N $N^{\circ} I I$

\begin{tabular}{|c|c|c|c|c|c|c|c|}
\hline Lot & Race & $A g \mathrm{e}$ & Alimentation & $\begin{array}{l}\text { Poids vif } \\
\text { départ } \\
(\mathrm{kg})\end{array}$ & $\begin{array}{l}\text { Poids vif } \\
\text { vide }(\mathrm{kg})\end{array}$ & $\begin{array}{l}\text { Différence } \\
(\Delta)(\mathrm{kg})\end{array}$ & $\begin{array}{c}\Delta / \text { Poids vif } \\
\text { depart } \\
\text { (p.100) }\end{array}$ \\
\hline $\mathrm{zHI}$ & z.M. & 4 a $9 m$ & Herbe & 311 & 250,5 & 60,5 & 19,4 \\
\hline $\mathrm{ZH} 2$ & Z.M. & $\mathrm{g} a$ & Herbe & 402,5 & 314,6 & $B 7,9$ & 21,8 \\
\hline $\mathrm{ZCl}$ & Z.M. & $19 \mathrm{~m}$ & $\begin{array}{l}\text { C.R. } \\
+ \text { foin }\end{array}$ & 329,8 & 280,8 & 49,0 & 14,8 \\
\hline $\mathrm{zC} 2$ & Z.M. & 3 a $3 m$ & $\begin{array}{l}\text { C.R. } \\
+ \text { foin }\end{array}$ & 356,3 & 324,2 & 32,2 & 9,0 \\
\hline $\mathrm{zC3}$ & $\mathrm{Z} . \mathrm{M}$. & $4 a$ & $\begin{array}{l}\text { C.R. } \\
+ \text { foin }\end{array}$ & 341 & 286,8 & 54,2 & 15,9 \\
\hline $\mathrm{zC} 4$ & Z.M. & $4 a$ & $\begin{array}{l}\text { llerbe } \\
+ \text { manioc }\end{array}$ & 352 & 302 & 50 & 14,2 \\
\hline $\mathrm{zC5}$ & $z . M$. & $4 a$ & $\begin{array}{l}\text { C.V. + peu } \\
\text { de foin }\end{array}$ & 368 & 327 & 41,0 & 11,1 \\
\hline $\mathrm{zC6}$ & $2 . \mathrm{M}$ & 7 a $7 \mathrm{~m}$ & $\begin{array}{l}\text { C.R. } \\
+ \text { foin }\end{array}$ & 354,4 & 292,6 & 61,8 & 17,4 \\
\hline $\mathrm{zC7}$ & 2.M. & 8 a $6 m$ & $\begin{array}{l}\text { C.R. } \\
+ \text { foin }\end{array}$ & 369,7 & 308,2 & 61,5 & 16,6 \\
\hline $\mathrm{zCB}$ & Z.M. & 9 a 37 & $\begin{array}{l}\text { C.R. } \\
+ \text { foin }\end{array}$ & 410,6 & 348,5 & 62,1 & 15,1 \\
\hline MH1 & $1 / 2 B$ & 3 a $6 m$ & Herbe & 443,2 & 343,5 & 99,7 & 22,4 \\
\hline $\mathrm{MH} 3$ & $1 / 2 \mathrm{~B}$ & 4 a $5 \mathrm{~m}$ & Herbe & 401,0 & 317,7 & 83,3 & 20,7 \\
\hline MH5 & $1 / 2 B$ & 5 a $6 \pi$ & Herbe & 591,5 & 484,3 & 107,2 & 18,1 \\
\hline $\mathrm{MC} 2$ & $5 / 8 \mathrm{~B}$ & $22 m$ & $\begin{array}{l}\text { C.R. } \\
+ \text { foin }\end{array}$ & 398,0 & 349,6 & 48,4 & 12,1 \\
\hline
\end{tabular}

C.R. = concentrě ratjonné; Z.M. = zébu Malgache;

C.V. = concentrë à volontë; $\quad 1 / 2 \mathrm{~B}=1 / 2$ Brahman.

\section{Influence de l'âge}

Les lots $\mathrm{MH}_{1}$ ( 3 ans 6 mois) et $\mathrm{MH}_{\text {, }}(5$ ans 6 mois) sont des métis $1 / 2$ Brahman élevés à l'herbe; les pourcentages de contenus digestifs par rapport au poids vif départ sont resfectivement 22,4 p. 100 et 18,1 p. 100 .
Les lots $\mathrm{ZC}_{i 3}$ ( 7 ans 7 mois), $\mathrm{ZC}_{\mathrm{i}}$ ( 8 ans 6 mois) et $\mathrm{ZC}_{8}$ (9 ans 3 mois) sont des Zébus Malgaches finis au concentré rationné pendant quatre mois, les pourcentages des contenus digestifs sont respectivement 17,4 p. 100 , 16,6 p. 100 et 15,1 p. 100. 
Il semble donc, au vu de ces deux comparaisons que le pourcentage de poids de contenu digestif diminue avec l'âge des animaux au moins pour des animaux ayant été élevés toute leur vie ou une grande partie de leur vie à l'herbe; il faut noter que le facteur âge est associé au facteur poids.

\section{Influence du mode d'alimentation}

Les lots $\mathrm{ZC}_{8}$ (finition au concentré) et $\mathrm{ZH}_{2}$ (herbe) sont des Zébus Malgaches d'âges et de poids voisins; les pourcentages des contenus digestifs sont respectivement 15,1 p. 100 et 21,8 p. 100.

Les lots $\mathrm{ZC}_{4}$ (herbe + manioc) et $\mathrm{ZH}_{1}$ (herbe) sont des Zébus Malgaches d'âges voisins; les pourcentages sont respectivement 14,2 p. 100 et 19,4 p. 100.

On constate une différence très importante des poids des contenus digestifs entre les animaux recevant une forte proportion de concentré dans la ration et ceux recevant uniquement du fourrage grossier. Dans les exemples précités la différence de pourcentage est de l'ordre de 6 p. $100 \mathrm{du}$ paids vif au départ.

La proportion d'aliment concentré dans la ration détermine aussi une différence de poids de contenu digestif ainsi qu'en témoigne la comparaison entre le lot $\mathrm{ZC}_{5}$ de 4 ans engraissé au concentré ad libitum pendant 6 mois (le concentré étant l'essentiel de leur ration) et le lot $\mathrm{ZC}_{3}$ de 4 ans engraissé au concentré rationné pendant 6 mois, du foin entrant pour une part non négligeable de la ration : les pourcentages des contenus digestifs sont respectivement 11,1 p. 100 et 15,9 p. 100.

La durée de l'alimentation concentrée est étudiée en comparant le lot $\mathrm{ZC}_{3}$, déjà cité, d'animaux de 4 ans, engraissés au concentré rationné plus foin pendant 6 mois, avec le lot $\mathrm{ZC}_{2}$ de 3 ans 3 mois, engraissé au concentré rationné plus foin, pendant 12 mois : les pourcentages de contenus digestifs sont respectivement 15,9 p. 100 et 9,0 p. 100 .

On constate un poids de contenu digestif nettement inférieur chez les animaux engraissés pendant 1 an au concentré rationné. Il faut noter que l'engraissement intensif a, ici, commencé alors que le lot $\mathrm{ZC}_{2}$ était plus jeune que le lot $\mathrm{ZC}_{4}$, ce qui a pu contribuer à un moindre développement des réservoirs gastriques.

\section{Influence de la race}

Les lots $\mathrm{ZH}_{2}$ (Zébu Malgache) et $\mathrm{MH}_{1}$ (1/2 Brahman) sont des animaux d'herbe présentant un état d'engraissement correct; les pourcentages de contenus digestifs sont respectivement 21,8 p. 100 et 22,4 p. 100 .

Les lots $\mathrm{ZH}_{1}$ (Zébu Malgache) et $\mathbf{M H}_{;}$ (1/2 Brahman) sont des animaux d'herbe présentant un état insuffisant; les pourcentages respectifs sont 19,4 p. 100 et 20,7 p. 100 .

Les lots $\mathrm{ZC}_{1}$ (Zébu Malgache), $\mathrm{ZC}_{2}$ (Zébu Malgache) et $\mathrm{MC}_{\text {. ( }}(5 / 8 \mathrm{Brahman})$ ont été finis au concentré rationné; les pourcentages sont respectivement 14,8 p. $100,9,0$ p. 100 et 12,1 p. 100.

On constate, aussi bien pour les animaux d'herbe que pour les animaux ayant subi une finition, qu'il n'apparaît pas de différence raciale nette entre les Zébus Malgaches et les métis Brahman; il faut noter cependant qu'il s'agit de deux races de Zébus, ce qui peut expliquer cette concordance des résultats.

\section{Conclusions}

On notera tout d'abord qu'il existe des interaotions entre le facteur poids et certains facteurs étudiés puisque le poids croît avec l'âge, avec l'alimentation concentrée et avec le degré de métissage.

Cependant des observations peuvent être faites.

Tout d'abord on constate la variation très élevée du poids des contenus digestifs de 32,2 à $114,3 \mathrm{~kg}$ pour les lots extrêmes et, en pourcentage du poids vif de 9,0 p. 100 à 22,4 p. 100 , soit une variation supérieure au doublement. Ces variations sont d'une grande importance économique et expliquent en partie la préférence accordée par les bouchers à certains types d'animaux (par exemple ceux engraissés au concentré) puisque pour un poids vif de $400 \mathrm{~kg}$ un taurillon précoce contiendra au départ $53 \mathrm{~kg}$ de produit strictement inutilisable de moins qu'un animal d'herbe.

Le pourcentage des contenus digestifs diminue assez sensiblement quand l'âge augmente (ou quand le poids augmente, ces deux facteurs étant liés) les variations observées allant de 2 à 4 p. 100 du poids vif de départ. 
TABLEAU $\mathbb{N}^{\circ}$ III

\begin{tabular}{|l|c|}
\hline \multicolumn{1}{|c|}{ Alimentation } & $\begin{array}{c}\text { Contenu digestif en } \\
\text { p.100 du P.V. départ }\end{array}$ \\
\hline Herbe & 19,4 à 22,4 \\
\hline $\begin{array}{l}\text { Concentré rationné, } \\
\text { engraissement court }\end{array}$ & $14, \mathrm{~B}$ à 17,4 \\
\hline $\begin{array}{l}\text { Concentré rationné, } \\
\text { engra issement long } \\
\text { ou bien concentré } \\
\text { ad Zibitum }\end{array}$ & 9,0 à 14,8 \\
\hline
\end{tabular}

Il varie très largement avec le mode d'alimentation et les chiffres rassemblés au tableau IJI seront utiles dans toute étude des poids vifs d'animaux.

L'influence de la race n'est pas apparue nettement dans nos expériences, les comparaisons étant surtout effectuées entre Zébus de races différentes ou métisses.
C'est donc manifestement le mode d'alimentation qui est déterminant, ce qui confirme les résultats d'études citées par CRAPLET (3).

\section{B. REPARTITION DU POIDS DU CONTENU DIGESTIF EN SES DEUX PARTIES}

Nous avons vu que le poids du contenu digestif présentait une importance économique à deux phases successives. Une partie en est éliminée entre le départ de la ferme et le moment de l'abattage; la différence entre le poids vif au départ et le poids vif avant abattage lui est pour sa quasi-totalité imputable. Une autre partie est encore présente dans l'animal au moment de l'abattage et influence beaucoup le rendement sur poids vif avant abattage (tableau IV).

TABLLAU N $\mathrm{N}^{\mathrm{T}} \mathrm{IV}$

\begin{tabular}{|c|c|c|}
\hline & $\begin{array}{c}\text { Contenu digestif } \\
\text { perdu avant } \\
1 \text { 'abatcage (p.100) }\end{array}$ & $\begin{array}{c}\text { Contenu digestí } \\
\text { restant à } \\
\text { l'abatcağ (p.100) }\end{array}$ \\
\hline $\begin{array}{l}\text { Miadanu herbe } \\
\text { (16 animaux) }\end{array}$ & 67,6 & 32,4 \\
\hline $\begin{array}{l}\text { Ki dnj asod herbe } \\
\text { (40 an imalus.) }\end{array}$ & 49.4 & 50,6 \\
\hline $\begin{array}{l}\text { Miadana concentré } \\
(10 \text { animaux })\end{array}$ & 72,0 & 28,0 \\
\hline $\begin{array}{l}\text { kianjasod concentre } \\
(65 \text { animaux })\end{array}$ & 51,8 & 48,2 \\
\hline $\begin{array}{l}\text { Tananardve concentré } \\
\text { (9 animax) }\end{array}$ & 43,6 & 56,4 \\
\hline
\end{tabular}

Les animaux étudiés ont tous été abattus à Tananarive et provenaient de trois sources différentes :

- Laboratoire de Tananarive: le transport était alors très réduit $(1 / 2$ heure de camion, et le jeûne était d'une quinzaine d'heures).

- Du Centre de Kianjasoa, situé à $180 \mathrm{~km}$ de l'abattoir; le transport durait de cinq à six heures de camion et le jeûne de 15 à 24 heures.

- Du Centre de Miadana, situé à $520 \mathrm{~km}$ de l'abattoir; le transport en camion durait quinze heures environ et le jeûne de 30 à 48 heures.
La durée de jeûne influence logiquement la quantité de contenu digestif perdue entre le départ de la ferme et le lieu d'abattage, et cela d'une façon très importante; pour les animaux d'herbe, il ne reste plus que 32,4 p. 100 du contenu initial pour les animaux de Miadana alors qu'il en reste encore 50,6 p. 100 pour les animaux de Kianjasoa. Pour les animaux engraissés au concentré les proportions restantes sont respectivement de 28,0 p. 100 et 48,2 p. 100 et 56,4 p. 100 pour les animaux de Tananarive. On peut remarquer que la vidange du tube digestif est assez rapide, puisque la moitié est expulsée en moins de 24 heures; le stress que subissent les animaux au cours de leur 
voyage en camion, fait de peur et «mal de voiture ", accélère certainement cette vidange.

Le rendement vrai (ou rendement sur poids vifs vide) est le plus intéressant puisqu'il rend compte de l'impontance relative de la carcasse et du cinquième quartier.

Le rendement commercial (ou rendement sur poids vif départ) est plus synthétique puisqu'il rend compte de l'importance relative de la carcasse par rapport à l'ensemble. (Cinquième quantier plus contenus digestifs compris.) Il est par contre plus difficile d'interprétation puisqu'on connaît avec imprécision le poids du contenu digestif.

Le rendement "à jeun" (ou rendement sur poids vif avant abattage) est sujet à interprétation erronée de la valeur des animaux; on voit par exemple que les animaux de Miadana seront systématiquement avantagés par rapport aux autres. La seule raison de s'y référer est son emploi fréquent dans certains abattoirs.

\section{POIDS DE CARCASSES ET RENDEMENTS}

Dans les résultats donnés ci-dessous, la carcasse comprend les rognons (chair et graisse) la queue, l'onglet et la hampe, la totalité de la graisse sous-cutanée et la bosse.

Les résultats d'un lot de castrés Charollais contrôlés à Jouy en Josas [LE GUELTE, 1964 (9)] sont cités afin de pouvoir établir des comparaisons avec les meilleurs animaux européens.

\section{A. LE ZEBU MALGACHE TRADITIONNEL}

Quatre lots ont été contrôlés qui permettront, à défaut d'une étude exhaustive, de cerner les caractéristiques de carcasses du Zébu Malgache élevé selon les méthodes habituelles; ils comprennent deux lots élevés à l'herbe uniquement (le lot $\mathrm{ZH}_{1}$, non fini et le lot $\mathrm{ZH}_{2}$ bien fini), un lot recevant du manioc vent en fin de carrière en plus de l'herbe $\left(\mathrm{ZC}_{4}\right)$ et un lot de "bœuf de fosse ", engraissement intensif traditionnel décrit par SERRES (14) (lot $\mathrm{ZC}_{9}$ ), (tableau V).
Le lot le plus intéressant est $\mathrm{ZH}_{2}$, qui représente la première qualité pour le pays; il s'agit d'animaux d'extensif de 7 à 10 ans, de $400 \mathrm{~kg}$ de poids vif environ, en état de gras satisfaisant; on constate que le poids des carcasses approche les $200 \mathrm{~kg}$, le rendement commercial est de 48,3 p. 100 et le rendement vrai 61,8 p. 100 .

Le lot $\mathrm{ZH}_{1}$ donne un poids de carcasses très insuffisant $(141,6 \mathrm{~kg})$ un rendement commercial de 45,5 p. 100 et un rendement vrai de 56,5 p. 100 ; il représente la qualité dite " de fabrication », animaux de 4 à 7 ans, trop maigres.

Ces deux lots sont un bon échantillonnage des deux types d'animaux abattus à Tananarive, puisque les statistiques de la SEVIMA portant sur un grand nombre d'animaux indiquent que les rendements commerciaux varient de 39 à 51 p. 100.

Le lot $\mathrm{ZC}_{4}$, fini avec distribution de manioc, en plus du pâturage, présente une amélioration assez nette par rapport au lot $\mathrm{ZH}_{1}$ sensiblement de même âge, avec des carcasses de $176,8 \mathrm{~kg}$, un rendement commercial de 50,2 p. 100 et un rendement vrai de 58,5 p. 100 ; il représente les animaux finis traditionnellement au manioc sur les pourtours des Hauts Plateaux, âgés de 4 ans et demi à 8 ans et qui, au point de vue qualité, s'étalent entre ce lot $\mathrm{ZC}_{4}$ et le lot $\mathrm{ZH}_{1}$.

Le lot $\mathrm{ZC}_{9}$, ou bœuf de fosse, est une spéculation très particulière de la région des Hauts Plateaux, animaux âgés subissant un engraissement intensif de dix mois et qui deviennent très gras; les carcasses sont ici de $269 \mathrm{~kg}$, le rendement commercial de 59,2 p. 100 et le rendement vrai de 66,7 p. 100 ; ces résultats sont le maximum de ce que l'on peut atteindre avec le Zébu Malgache par une finition intensive sur des animaux choisis avec soin par des éleveurs compétents; elles démontrent que la race répond à une alimentation intensive; on recherche une adaptation de cette technique pour la finition industrielle économique.

Ces résultats nous permettent de définir une norme des rendements vrais de la race en fonction de la qualité de carcasse (état viande et gras), (tableau VI).

Nous sommes assez loin des normes du bétail européen des races à viande, ce qui indique que la "conformation bouchère " du Zébu Malgache n'est pas des meilleures. 
TABLEAU $\mathrm{N}^{\circ} \mathrm{V}$

Zébu Vialgache trađitionnel

\begin{tabular}{|c|c|c|c|c|}
\hline$L \circ t$ & $2 \mathrm{HL}$ & $\mathrm{ZH} 2$ & $\mathrm{zC4}$ & $2 \mathrm{Cg}$ \\
\hline$A g e$ & 4 a $9 \mathrm{~m}$ & 8 ans & 4 ans & 12 an 5 \\
\hline Alimentation & Herbe & Herbe & Herbe + man1oc & Herbe + maniac \\
\hline Poids vif dêpart $(\mathrm{kg})$ & 311 & 402,5 & 352 & 454 \\
\hline Foids carcasse chaude $(\mathrm{kg})$ & 144,3 & 198,6 & & 274,1 \\
\hline Poids carcasse froide ( $\mathrm{kg}$ ) & 141,6 & 194,6 & 176,8 & 269,0 \\
\hline Renaement commercial (p.100) & 45,5 & 48,3 & 50,2 & 59,2 \\
\hline Rendement à jeur $(p .100)$ & 49,7 & 54.4 & 54,9 & 62,5 \\
\hline Rendement vrai (p.100) & 56,5 & 61,8 & 58,5 & 66,7 \\
\hline Longueur $(\mathrm{cm})$ & 133,3 & 122,3 & 113,0 & 125,0 \\
\hline Indice ëtat viande & 1,25 & 1,59 & 1,56 & 2,15 \\
\hline Epaisseur cuisse (cm) & 19,7 & 22,8 & 22,3 & 28,0 \\
\hline$\frac{\text { Epaisseur cuisse }}{\text { longueur }}(p .100)$ & 17,3 & 18,6 & 19.7 & 22,4 \\
\hline Gras de rognons $(\mathrm{kg})$ & 1,50 & 2,73 & 3,0 & 6,20 \\
\hline$\frac{\text { Gras de rognon }}{\text { carcasse froide }}$ (p.loo) & 1,06 & 1,40 & 1,69 & 2,3 \\
\hline Rendement muscle (p.100) & 59 & 61,9 & 60,7 & \\
\hline Qualité & 2òme faible & 1 ère & lère faible & $\begin{array}{l}\text { inclassable } \\
\text { (très gras) }\end{array}$ \\
\hline
\end{tabular}

L'indice d'état de viande est ici le rapport du poids de carcasse froide, avec rognons (chair et graisse) et queue, à la langueur en $\mathrm{cm}$.

TABLEAL $\mathbb{N}^{\mathrm{V}} \mathrm{VI}$

\begin{tabular}{|c|c|c|c|c|c|}
\hline E t a t & Insuffısant & Correct & Bon & Très bon & $\begin{array}{c}\text { Castrés } \\
\text { Charollais }\end{array}$ \\
\hline $\begin{array}{l}\text { Rendement vrai } \\
(\mathrm{p}, 100)\end{array}$ & $<58$ & 58 à 61 & 61 à 63 & $>63$ & 68,5 \\
\hline
\end{tabular}

\section{B. ZEBUS MALGACHES NOURRIS EN « FEED LOT »}

Des essais de finition intensive ont été et sont actuellement pratiqués sur des Zébus Malgaches à différents âges. Le concentré distribué est constitué soit d'un mélange maïs, tourteau d'arachides, poudre d'os, soit d'un mélange équivalent à base de son de riz.

La durée de la finition est, bien entendu, plus longue pour les jeunes animaux que pour les vieux.

Les poids de carcasses obtenus sont assez variables puisqu'ils dépendent de deux fac- teurs : ils augmentent à la fois avec l'âge des animaux et avec la durée et l'intensité de l'alimentation; or, dans nos expériences, les animaux les plus jeunes ont subi l'alimentation la plus longue. Les lots jeunes $\mathrm{ZC}_{2}$ et $\mathrm{ZC}_{\bar{\pi}}$ ont subi une embouche coûteuse mais ont donné des carcasses lourdes (plus de $200 \mathrm{~kg}$ ) et de très bonne qualité (rendement vrai 63,7 et 66,4 p. 100). Le lot $\mathrm{ZC}_{3}$, jeune, ayant reçu une alimentation rationnée de 6 mois seulement, a donné des carcasses un peu légères $(179 \mathrm{~kg})$ mais de bonne qualité (rendement vrai $62,5 \mathrm{p}$. 100). Le lot $\mathrm{ZC}_{1}$, très jeune, ayant subi une embouche coûteuse (10 mois) a donné des carcasses encore plus légères $(172 \mathrm{~kg}$ ) présentant un état musculaire correct et un état de gras 


\section{TABLEAU $N^{\circ} \mathrm{VII}$}

Zêbus Malgaches nourris en "feed lot"

\begin{tabular}{|c|c|c|c|c|c|c|c|}
\hline$L \circ t$ & $2 \mathrm{Cl}$ & $z \mathrm{C} 2$ & $\mathrm{zC3}$ & $2 \mathrm{C5}$ & 2c6 & $2 \mathrm{C} 7$ & $2 \mathrm{CB}$ \\
\hline A $g$ e & 19 mois & 3 a $3 \mathrm{~m}$ & 4 ans & 4 ans & 7 a $7 \mathrm{~m}$ & 8 a $6 \mathrm{~m}$ & 9 a $3 \mathrm{~m}$ \\
\hline Alimentation & $\begin{array}{l}\text { C.R. } \\
\text { I0 mois }\end{array}$ & $\begin{array}{c}\text { C.R. } \\
12 \text { mois }\end{array}$ & $\begin{array}{l}\text { C.R. } \\
6 \text { mois }\end{array}$ & $\begin{array}{c}\text { C.V. } \\
6 \text { mois }\end{array}$ & $\begin{array}{l}\text { C.R. } \\
3 \text { mois }\end{array}$ & $\begin{array}{l}\text { C.R. } \\
3 \text { mois }\end{array}$ & $\begin{array}{l}\text { C.R. } \\
3 \text { molis }\end{array}$ \\
\hline Poids vif départ $(\mathrm{kg})$ & 330,0 & 356,3 & 341 & 368 & 354,4 & 369,7 & 410,6 \\
\hline Poids carcasse chaude $(\mathrm{kg})$ & 174,2 & & 180,6 & 223,0 & 179,0 & & \\
\hline Poids carcasse froide (kg) & 171,9 & 206.7 & 179,2 & 217,4 & 173,8 & 180,0 & 216,3 \\
\hline Rendement commercial (p.100) & 52,1 & 58,0 & 52,5 & 59,0 & 49,0 & 48,7 & 52,6 \\
\hline Rendement à jeûn (p.100) & 58,3 & 61,5 & 57,5 & 63,3 & 53,2 & 52,1 & 56,3 \\
\hline Rendement vrai (p.100) & 61,2 & 63,7 & 62,5 & 66,4 & 59,4 & 58,4 & 62,0 \\
\hline Longueur $(\mathrm{cm})$ & 108,2 & 110,8 & 111,0 & 111,5 & 118,7 & 120,4 & 122,4 \\
\hline Indice état viande & 1,58 & 1,86 & 1,61 & 1,95 & 1,46 & 1,49 & 1,77 \\
\hline Epaisseur cuisse (cm) & 21,1 & 22,6 & 21,1 & $2 I, 9$ & 21,6 & 21,6 & 22,9 \\
\hline$\frac{\text { Epaisseur cuisse }}{\text { longueur }}(\mathrm{p} .100)$ & 19,5 & 20,4 & 19,0 & 19,6 & 18,2 & 17,9 & 18,7 \\
\hline Gras de rognons $(\mathrm{kg})$ & 2,56 & 8,9 & 3,88 & 8,35 & 1,57 & 1,90 & 3,28 \\
\hline$\frac{\text { Gras de rognons }}{\text { carcasse froide }} \quad(p .100)$ & 1,48 & 4,3 & 2,2 & 3,8 & 0,90 & 1,05 & 1,48 \\
\hline Rendement muscle (p.100) & 58 & 57,2 & 59 & 58,4 & 57,2 & 58,3 & \\
\hline Qualitë & $\begin{array}{l}2 \text { ème } \\
\text { moyerne }\end{array}$ & $\begin{array}{l}2 \text { ème } \\
\text { normale }\end{array}$ & $\begin{array}{l}2 \text { ème } \\
\text { forte }\end{array}$ & $\begin{array}{l}2 \text { ème } \\
\text { normale }\end{array}$ & $\begin{array}{l}2 \text { ène } \\
\text { forte }\end{array}$ & $\begin{array}{l}1 \text { ère } \\
\text { faible }\end{array}$ & $\begin{array}{l}1 \text { ère } \\
\text { faible }\end{array}$ \\
\hline
\end{tabular}

un peu insuffisant dû sans doute à leur âge (rendement vrai 61,2 p. 100).

Les trois lots d'animaux âgés $\left(\mathrm{ZC}_{6}, \mathrm{ZC}_{7}\right.$ et $\mathrm{ZC}_{8}$ ont subi une finition peu onéreuse. Les deux lots $\mathrm{ZC}_{6}$ et $\mathrm{ZC}_{7}$ ont donné des résultats de boucherie insuffisants : ils ont été abattus trop tôt et auraient été justiciables de un ou deux mois supplémentaires de finition pour atteindre des rendements satisfaisants, qui ont été, par contre, atteints avec le lot $\mathrm{ZC}_{8}$. (Carcasses de $216 \mathrm{~kg}$ et rendement vrai de 62 p. 100).

Cette série d'essais a permis d'affirmer qu'il était possible d'obtenir de bonnes carcasses avec le Zébu Malgache et de préciser un certain nombre de normes pour entreprendre les essais actuellement en cours, qui ont un objectif strictement économique.

\section{INFLUENCE DE LAMELIORATION GENETIQUE SEULE}

Nous n'envisagerons ici l'amélioration du
Zébu Malgache que par croisement avec le Brahman du Texas.

Nous étudierons d'abord les résultats de ces métis élevés uniquement sur pâturage naturel.

On constate tout d'abord une supériorité considérable en poids vifs par rapport aux Zébus Malgaches de même âge, due à une croissance plus rapide. Les poids de carcasses sont en conséquence directe, supérieurs.

Les rendements vrais sont tous supérieurs à 60 p. 100 , même pour les deux lots provenant de Miadana qui vivent dans des conditions plus difficiles et qui étaient en état de gras insuffisant pour le lot $\mathrm{MH}_{4}$ (rendement vrai de 62,6 p. 100 cependant) et à la fois en état de muscle et en état de gras insuffisants pour le $\mathrm{MH}_{3}$ (60,5 p. 100 cependant). Les quatre autres lots dépassent 64 p. 100 de rendement vrai.

Il est incontestable que pour la "qualité bouchère " prise dans un sens large, les métis sont nettement supérieurs aux animaux de race locale. 
TABLEAU $\mathrm{N}^{\circ}$ VIII

Zébus amêliorês gểnétiquement

\begin{tabular}{|c|c|c|c|c|c|c|}
\hline$L \circ t$ & SHY1 & MH3 & MH5 & MH2 & MHA & 8H6 6 \\
\hline A $g$ e & 3 a $6 \mathrm{~m}$ & 4 a $6 \mathrm{~m}$ & 5 a $6 \mathrm{~m}$ & 3 a $6 \mathrm{~m}$ & 4 a $9 \mathrm{~m}$ & 5 a $6 \mathrm{~m}$ \\
\hline $\mathrm{R}$ a $c \mathrm{e}$ & $1 / 2 \mathrm{~B}$ & $1 / 2 \mathrm{~B}$ & $1 / 2$ B & $3 / 4 B$ & $3 / 4 \mathrm{~B}$ & $3 / 4 \mathrm{~B}$ \\
\hline Alimentation & Herbe & Herbe & Herbe & Herbe & Herbe & Herbo \\
\hline Poids vif dêpart (kg) & 443,2 & 401,0 & 591,5 & 436,0 & 493,2 & 586,4 \\
\hline Poids carcasse chaude $(\mathrm{kg})$ & 226,3 & 196,3 & 320,6 & 233,5 & & 310,6 \\
\hline Poids carcasse froide (kg) & 221,8 & 192,3 & 314,0 & 228,8 & 243,1 & 304,8 \\
\hline Rendement cormercial (p.100) & 50,0 & 47,9 & 53,1 & 52,5 & 49.5 & 51,9 \\
\hline Rendement à jeûn $(p \cdot 100)$ & 56,5 & 54,9 & 57,8 & 57,6 & 58,5 & 57,9 \\
\hline Rendement vrai (p.100) & 64,5 & 60,5 & 64,8 & 65,3 & 62,6 & 64,5 \\
\hline Longueur (cra) & 123,8 & 122,3 & 136.9 & 121,8 & 126,4 & 136,0 \\
\hline Indice êtat viande & 1,79 & 1,57 & 2,29 & 1,87 & 1,92 & 2,24 \\
\hline Epaisseur cuisse $(\mathrm{cm})$ & 25,7 & 22,7 & 26,7 & 26,2 & 24,2 & 26,4 \\
\hline$\frac{\text { Epaigseur cuisse }}{\text { longueur }}$ (p.100) & 20,7 & 18,5 & 19,5 & 21,5 & 19,1 & 19,4 \\
\hline Gras de rognons ( $\mathrm{kg}$ ) & 2,89 & 0,77 & 5,50 & 1,90 & 4,0 & 3,55 \\
\hline$\frac{\text { Gras de rognons }}{\text { carcasse froide }}(p \cdot 100)$ & 1,3 & 0,4 & 1,7 & 0,8 & 1,6 & 1,1 \\
\hline Rendement muscle (p.100) & 64,1 & 63,6 & 64,2 & 64,7 & 62,2 & 63,1 \\
\hline Qualitë & $\begin{array}{c}\text { I ère } \\
\text { normale }\end{array}$ & $\begin{array}{c}1 \text { ère } \\
\text { normale }\end{array}$ & $\begin{array}{l}1 \text { ère } \\
\text { bonne }\end{array}$ & $\begin{array}{c}1 \text { ère } \\
\text { normale }\end{array}$ & $\begin{array}{l}1 \text { ère } \\
\text { faible }\end{array}$ & $\begin{array}{c}1 \text { ère } \\
\text { normale }\end{array}$ \\
\hline
\end{tabular}

\section{RESULTATS}

\section{DE L'AMELIORATION GENETIQUE ET DE L'ALIMENTATION}

Deux lots de jeunes animaux métis ont été finis au concentré, le lot $\mathrm{MC}_{2}$ de 5/8 Brahman pendant 6 mois et le lot $\mathrm{MC}_{1}$ de taurillons métis Frison-Zébu pendant 8 mois; la troisième colonne donne des résultats de castrés Charollais déjà cités, (tableau IX).

Les poids de carcasses dépassent largement les $200 \mathrm{~kg}$, ce qui était recherché. Les taurillons 1/2 Frisons avaient un état musculaire parfait et un état de gras légèrement insuffisant provenant peut-être de leur jeune âge ou du fait qu'ils étaient entiers ou d'une combinaison de ces deux causes; leur rendement vrai est de 62,7 p. 100.

Les 5/8 Brahman étaient en bon état à la fois pour le muscle et le gras et ont eu un rendement vrai de 65,5 p. 100.

L'engraissement intensif n'a pas apporté d'amélioration des rendements ou de l'état de gras par rapport aux autres métis élevés en herbage, sauf probablement pour la tendreté de la viande étudiée plus loin.

L'avantage, par contre primordial de l'engraissement, est l'abaissement de moitié de l'âge d'abattage.

\section{E. CONCLUSION}

Le tableau $\mathrm{X}$ regroupe les résultats de rendements obtenus avec les différents types d'animaux :

Les Zébus Malgaches, s'ils ne sont pas en bon état, donnent des rendements très mauvais (57 p. 100 de rendement vrai). Les rendements deviennent corrects pour des animaux assez âgés (6 à 8 ans) et disposant d'un pâturage naturel assez bon, ils atteignent alors 49 p. 100 de rendement commercial et 62 p. 100 de rendement vrai, chiffres qui peuvent être atteints par des animaux plus jeunes mais finis avec supplémentation de manioc. Les animaux 
TABLEAU $\mathrm{N}^{\circ} \mathrm{IX}$

Amélioration gênêtique et de l'alimentation des zêbus

\begin{tabular}{|c|c|c|c|}
\hline$L \circ t$ & MC1 & MC2 & Charollais \\
\hline$R$ a c e & $\begin{array}{c}1 / 2 \\
\text { Frison } / \text { Zëbu }\end{array}$ & 5/8 Brahman & Charollais \\
\hline $\mathrm{A} g \mathrm{e}$ & $18 \mathrm{~m}$ & $22 \mathrm{~m}$ & $15 \mathrm{~m}$ \\
\hline Alimentation & $\begin{array}{l}\text { C.R. } \\
8 \text { mois }\end{array}$ & $\begin{array}{l}\text { C.v. } \\
6 \text { mois }\end{array}$ & \\
\hline Poids vif dêpart (kg) & 390,2 & 398,0 & \\
\hline Poids carcasse chaude ( $\mathrm{kg}$ ) & 219,3 & 233,7 & 313,6 \\
\hline Poids carcasse froide $(\mathrm{kg})$ & 217,6 & 229,1 & 308,5 \\
\hline Rendement commercial (p.100) & 55,7 & 57,5 & \\
\hline Rendement à jeôn $\langle p .100\rangle$ & 58,6 & 61,5 & \\
\hline Rendement vrai (p.100) & 62,7 & 65,5 & 68,5 \\
\hline Longueur $\langle\mathrm{cm}\rangle$ & 117,4 & 116,8 & 125,0 \\
\hline Indice état viande & 1,85 & 1,96 & 2,82 \\
\hline Epaisseur cuisse $(\mathrm{cm})$ & 22,8 & 23,3 & 28,2 \\
\hline$\frac{\text { Epaisseur cuisse }}{\text { Iongueur }}(p .100)$ & 19,4 & 20,0 & 22,5 \\
\hline Gras de rognons (kg) & 4,7 & 6.37 & 6,61 \\
\hline$\frac{\text { Gras de rognons }}{\text { carcasse froide }}(p .100)$ & 2,2 & 2,8 & 2,14 \\
\hline Rendement muscle (p.100) & 59,1 & & 66,3 \\
\hline Qualite & 2ème normale & lère faible & \\
\hline
\end{tabular}

correctement finis au concentré améliorent nettement leur performance pour atteindre 63 et même 66 p. 100 de rendement vrai pour des bœufs très gras.

L'amélioration génétique apporte un progrès considérable puisque les rendements vrais ne descendent pas en dessous de 60 p. 100 pour des métis en mauvais état et atteignent aisément 65 p. 100 pour des animaux en bon état de viande mais pas trop gras.

Le Zébu Malgache est un animal de petit format dont les carcasses atteignent difficilement $200 \mathrm{~kg}$ sans risque de surcharge graisseuse. Cependant, avec l'évolution du marché mondial vers des formes de commercialisation en désossé-congelé et en lyophilisé, les seuils de poids de carcasses s'abaisseront probablement et ce caractère prendra moins d'importance.
TABLEAU $\mathrm{N}^{\circ} \mathrm{X}$

\begin{tabular}{|c|c|c|}
\hline T y p e & $\begin{array}{l}\text { Rendement } \\
\text { commercial }\end{array}$ & $\begin{array}{l}\text { Rendement } \\
\text { vrai (p.100) }\end{array}$ \\
\hline $\begin{array}{l}\text { Zëbu Malgache d'herbe } \\
\text { en mauvais état }\end{array}$ & 46 & 57 \\
\hline $\begin{array}{l}\text { Zébu Malgache d'herbe } \\
\text { en bon état }\end{array}$ & 49 & 62 \\
\hline $\begin{array}{l}\text { Métis } d^{\prime h} \text { herbe } \\
\text { en mauvais état }\end{array}$ & 48 & 60 \\
\hline $\begin{array}{l}\text { Métis d'herbe } \\
\text { en bon êtat }\end{array}$ & 53 & 65 \\
\hline $\begin{array}{l}\text { Zébu Malgache insuf- } \\
\text { Eisamment fini }\end{array}$ & 49 & 60 \\
\hline $\begin{array}{l}\text { Zêbu Malgache suf- } \\
\text { Eisamment fini }\end{array}$ & 58 & 63 \\
\hline $\begin{array}{l}\text { Zébu Malgache très } \\
\text { gras }\end{array}$ & 59 & 66 \\
\hline Mètis fini au concentre & 56 & 65 \\
\hline
\end{tabular}




\section{CARACTERISTIQUeS DE CARCASSES}

\section{A. LE ZEBU MALGACHE}

(Voir tableaux $\mathrm{V}$ et VII.)

\section{Observations visuelles}

$\mathrm{ZH}_{1}$ : profil ischio tarsien subconcave à concave; couverture insuffisante; graisse jaune, viande de bonne tenue; entrecôtes et avants faibles mais bonne présentation des cuisses.

$\mathrm{ZH}_{2}$ : profil droit à subconvexe; couverture correcte; graisse jaune à orange clair; bonne viande.

$\mathrm{ZC}_{4}$ : profil subconcave à droit; couverture un peu insuffisante; graisse jaune clair à jaune; belle présentation; comparable à la qualité New-Zeeland, mais manque de poids.

$\mathrm{ZC}_{9}$ : (Bouf de fosse) : profil convexe; couverture très excessive; graisse orange et très molle; viande très persillée et marbrée; surcharge de graisse considérable.

$\mathrm{ZC}_{1}$ : profil droit; couverture de assez bonne à un peu insuffisante; graisse blanche à jaune très clair.

$\mathrm{ZC}_{2}$ : profil droit en moyenne; couverture de correcte à excessive; graisse blanche à jaune, assez molle; viande de bon aspect avec un petit persillé, trop de gras interne; bonne épaisseur moyenne mais pas mûre.

$\mathrm{ZC}_{3}$ : profil droit à subconvexe; couverture de correcte à un peu insuffisante; graisse jaune clair.

$\mathrm{ZC}_{\overline{,}}$ : profil droit à convexe; couverture en excès sur certains animaux; graisse jaune clair à jaune, belle viande persillée.

$\mathrm{ZC}_{\mathrm{f}}$ : profil subconcave à rectiligne; couverture insuffisante à correcte; graisse jaune clair à orange; belle viande; belles cuisses mais entrecôtes faibles.

$\mathrm{ZC}_{7}$ : profil concave à subconcave; couverture moyenne; graisse jaune clair à orange; présentation correcte, belle viande; pas assez de gras interstitiel.

$\mathrm{ZC}_{8}$ : profil subconcave à rectiligne; couverture correcte; graisse jaune orange; belle viande.

\section{Longueur}

La mesure de la longueur est fidèle et facile à prendre. Il est apparu une liaison très forte entre la longueur de la carcasse et l'âge du lot.

TABLEAU $N^{\circ} \mathrm{XI}$

\begin{tabular}{|c|c|c|c|c|c|c|}
\hline \multicolumn{2}{|c|}{$L \circ \mathrm{t}$} & $\mathrm{ZC}_{1}$ & $2 C_{2}$ & $2 C_{3}$ & $\mathrm{zC}_{4}$ & $\mathrm{ZC}_{5}$ \\
\hline \multicolumn{2}{|l|}{ Age } & $20 \mathrm{~m}$ & $\beta$ a 3m & $4 a$ & $4 \mathrm{a}$ & $4 a$ \\
\hline \multicolumn{2}{|c|}{$\begin{array}{l}\text { Longueur } \\
(\mathrm{cm})\end{array}$} & 108,2 & 110,8 & 111,0 & 113,0 & 111,5 \\
\hline Lot & $\mathrm{ZH}_{1}$ & $2 C_{6}$ & $2 \mathrm{H}_{2}$ & $2 C_{7}$ & $2 C_{8}$ & $2 C_{9}$ \\
\hline Age & 4 a $9 \mathrm{~m}$ & a $7 \mathrm{~m}$ & $B a$ & 8 a $6 m$ & 9 a $3 m$ & $12 \mathrm{a}$ \\
\hline $\begin{array}{l}\text { Longueur } \\
\text { (cm) }\end{array}$ & 113,3 & 118,7 & 122,3 & 120,4 & 122,4 & 125,0 \\
\hline
\end{tabular}

\section{L'état de gras}

Il est mesuré ioi par le pourcentage de carcasse que représente le poids du gras des rognons. On l'appellera indice de gras.

On constate que cet indice varie très largement pour les 11 lots, de 0,90 p. 100 à 4,3 p. 100 . Il est particulièrement élevé pour les lots $\mathrm{ZC}_{2}$ et $\mathrm{ZC}_{\bar{i}}$, animaux assez jeunes ayant subi une finition au concentré à forte dose (concentré rationné pendant 12 mois et concentré à volonté pendant 6 mois). Les carcasses les plus grasses de toutes étaient celles des bœufs de fosse dont l'indice n'est que $2,3 \mathrm{p}$. 100. On sait en effet que le gras ne se dépose pas uniformément dans les différentes régions corporelles et qu'il commence par le gras interne; l'indice sera donc relativement peu élevé chez un animal vieux et très gras en raison de l'importance du gras de couverture, de la bosse et du gras interstitiel (le poids moyen de la bosse a atteint $17 \mathrm{~kg}$ pour le lot $\mathrm{ZC}_{9}$ ). Cependant, hormis ce cas extrême, on peut préciser la relation entre l'indice de gras et les observations visuelles de l'état de gras de la carcasse (tableau XII).

Il convient de remarquer que si un excès d'état de gras est presque toujours une pénalité pour les carcasses destinées à l'exportation, une 
TABLEAU N $N^{\circ} X I I$

Indices de gras

\begin{tabular}{|c|c|c|c|}
\hline Etat de gras & $\begin{array}{c}\text { Insuffisant } \\
(\mathrm{p} .100)\end{array}$ & $\begin{array}{c}\text { Correct } \\
\text { (p.100) }\end{array}$ & $\begin{array}{c}\text { Excès } \\
\text { (p.100) }\end{array}$ \\
\hline Animaux de moins de 4 ans & $<2$ & 2 à 3,5 & $>3,5$ \\
\hline Animaux de 4 ans à 6 ans & $<1,5$ & 1,5 à 2,5 & $>2,5$ \\
\hline Animaux de plus de 6 ans & $<1,1$ & 1,1 à 1,6 & $>1,6$ \\
\hline
\end{tabular}

insuffisance peut être bénéfique pour certaines présentation à la condition que l'état musculaire soit correct.

D'autre pant, un excès de graisse est bénéfique sur le marché inténieur en raison des habitudes alimentaires locales.

Ces précisions étant données, nous pouvons remarquer qu'un état de gras insuffisant est apparu pour le lot $\mathrm{ZH}_{1}$, boufs d'herbe de 4 ans 9 mois; cette observation, jointe à un poids de carcasse bien trop faible $(141,6 \mathrm{~kg})$ devrait condamner l'abattage de bœufs d'herbe de moins de 5 ans. Le lot $\mathrm{ZC}_{1}$, de 20 mois, fini au concentré pendant 10 mois, manque un peu d'état de gras; son jeune âge en est certainement la cause. Le lot $\mathrm{ZC}_{3}$ de 4 ans au concentré rationné pendant 6 mois manque un peu de couverture ainsi que le lot $\mathrm{ZC}_{6}$, de 7 ans 7 mois au concentré rationné pendant 3 mois; pour ce dernier dot, il est probable qu'un mois supplémentaire de finition aurait été bénéfique. $\mathrm{Si}$ l'on compare aux lots $\mathrm{ZC}_{7}$ et $\mathrm{ZC}_{8}$ un peu plus âgés, il semble que l'utilisation d'animaux de plus de 8 ans soit un bon point pour obtenir un engraissement correct dans le cadre d'une finition courte.

Trois lots ont atteint un excès d'état de gras : le lot $\mathrm{ZC}_{9}$ (bœuf de fosse); te lot $\mathrm{ZC}_{2}$ qui concerne des animaux jeunes ( 3 ans 3 mois) ayant subi une finition longue car le but de l'expérience était d'obtenir des carcasses de $200 \mathrm{~kg}$; cette limite de poids a été atteinte au prix d'un excès de graisse et il ne semble pas qu'il faille viser ce poids avec des Zébus Malgaches jeunes, dans une optique de finition industrielle économique; le lot $\mathrm{ZC}_{5}$ de 4 ans avec concentré à volonté pendant 6 mois était trop gras car l'expérience consistait en la recherche de l'augmentation maximale de poids; il n'est aucunement étonnant d'avoir obtenu des carcasses trop grasses.

\section{Epaisseur de la cuisse et indice de muscle}

L'épaisseur de la cuisse, prise à la sonde, est un bon indice de développement musculaire bien qu'une erreur intervienne par le fait qu'un animal très gras présente du gras interstitiel et de couverture qui augmente un peu la mesure. Les valeurs vont de 19,7 à 28 centimètres; l'indice de muscle employé est le pourcentage de la longueur représenté par l'épaisseur de la cuisse, indiquant si, pour sa taille, l'animal est musclé ou non; les valeurs vont de 17,3 p. 100 à 22,4 p. 100 . On peut comparer à l'indice de gras:

TABLEAU $N^{\circ} \mathrm{XIII}$

\begin{tabular}{|c|c|c|c|c|c|c|c|c|c|c|c|}
\hline$L \quad \circ t$ & $\mathrm{ZH}_{1}$ & $2 \mathrm{C}_{7}$ & $2 C_{6}$ & $2 \mathrm{Hl}_{2}$ & $\mathrm{ZC}_{\mathrm{B}}$ & $\mathrm{zC}_{3}$ & $\mathrm{zC}_{1}$ & $\mathrm{zC}_{5}$ & $\mathrm{ZC}_{4}$ & $\mathrm{zC}_{2}$ & $\mathrm{zC}_{g}$ \\
\hline $\begin{array}{l}\text { Indice de } \\
\text { muscle (p. 100) }\end{array}$ & 17,3 & 17,9 & 18,2 & $1 B, 6$ & 18,7 & 19,0 & 19,5 & 19,6 & 19,7 & 20,4 & 22,4 \\
\hline $\begin{array}{l}\text { Indice de } \\
\text { gras }\end{array}$ & 1,0 & 1,0 & 0,9 & 1,4 & 1,5 & 2,2 & 1,5 & $3, B$ & 1,7 & 4,3 & 2,3 \\
\hline
\end{tabular}

Les animaux les plus gras sont aussi les plus musclés; le lot $\mathrm{ZC}_{4}$ dont l'indice de gras est faible fait exception, mais il est apparu effectivement à l'observation visuelle et à la découpe comme bien musclé et peu gras. Une autre exception est le lot $\mathrm{ZC}_{1}$, les plus jeunes des animaux finis au concentré, qui ont un bon indice musculaire et un indice de gras faible. 


\section{Indice d'état de viande}

C'est le rapport du poids de carcasse ressuyée à la longueur. Il représente, en quelque sorte, la synthèse des deux précédents indices puisqu'un animal musclé et gras aura, pour sa longueur, un poids élevé et l'on a vu que les indices de gras et de muscle étaient liés.

Les valeurs se répartissent entre 1,25 et 2,15; si l'on excepte les trois lots qui étaient nettement trop gras $\left(\mathrm{ZC}_{9}, \mathrm{ZC}_{2}\right.$ et $\left.\mathrm{ZC}_{5}\right)$, les valeurs vont de 1,25 à 1,77 , ce qui, comparé au 2,82 des bouvillons Charollais, indique que le Zébu Malgache a une conformation bouchère relativement médiocre; des indices d'état de viande de 2 ne peuvent être atteints que par une surcharge graisseuse; ils ne sont donc pas à rechercher.

A degré d'engraissement comparable, l'indice augmente avec l'âge, ce qui est logique puisque le tissu osseux se développe avant les tissus musculaire et graisseux.

L'indice d'état de viande, intra race, est en liaison étroite avec le rendement vrai comme le montre le tableau suivant où les indices sont disposés en ordre croissant :

TABLEAU N $\mathrm{N}^{\circ} \mathrm{XIV}$

\begin{tabular}{|c|c|c|c|c|c|c|c|c|c|c|c|}
\hline $\mathrm{L} \circ \mathrm{t}$ & $\mathrm{zll}_{1}$ & $\mathrm{zC}_{6}$ & $\mathrm{zC}_{7}$ & $\mathrm{zC}_{4}$ & $\mathrm{zC}_{1}$ & $\mathrm{zI}_{2}$ & $\mathrm{zC}_{3}$ & $\mathrm{zC}_{8}$ & $\mathrm{zC}_{2}$ & $\mathrm{zC}_{5}$ & $\mathrm{zC}_{9}$ \\
\hline $\begin{array}{l}\text { Indice } \\
(\mathrm{p} \cdot 100)\end{array}$ & 1,25 & 1,46 & 1,49 & 1,56 & 1,58 & 1,59 & 1,61 & 1,77 & 1,86 & 1,95 & 2,15 \\
\hline $\begin{array}{l}\text { Rendement } \\
\text { vrai }\end{array}$ & 56,5 & 59,4 & 58,4 & 58,5 & 61,2 & 61,8 & 62,5 & 62,0 & 63,7 & 66,4 & 66,7 \\
\hline
\end{tabular}

Cette liaison apparaît intéressante car la mesure du rendement vrai est peu pratique à l'échelon industriel alors que la mesure de la longueur est très rapide.

\section{Rendement en muscle}

Pour 9 lots sur 11, le rendement en muscle de la carcasse, après désossage et parage a été effectué. Les valeurs vont de 57,2 p. 100 à 61,9 p. 100 , ce qui est faible.

Le pourcentage de gras est le facteur principal du rendement en muscle, plus le gras est développé, moins le rendement en muscle est élevé.

Le développement musculaire influe aussi, mais d'une façon bien moindre, sur le rendement musculaire.

L'indice de gras et l'indice de muscle agissent donc en sens contraire sur le rendement en muscle, et nous avons vu qu'ils variaient dans le même sens. En conséquence, pour nos lots qui présentent une grande variabilité de races. d'âges et de modes d'alimentation, le rendement en muscle n'est lié d'une façon simple à aucun des indices précités (l'épaisseur de la cuisse, à poids de carcasse constant n'a pas présenté, non plus, de liaison nette avec le rendement en muscle). Des prévisions de détail peuvent être effectuées, mais, dans l'ensemble, aucune mesure ne remplace le désossage-parage réel de la carcasse, pour obtenir le rendement en muscle.

\section{B. LES ANIMAUX METISSES \\ (Voir tableaux VIII et IX)}

\section{Observations visuelles}

$\mathrm{MH}_{1}$ : profil subconvexe à convexe, couverture de graisse correcte, graisse jaune clair à jaune.

$\mathrm{MH}_{3}$ : subconcave à concave; couverture totalement insuffisante; graisse jaune clair à jaune; belle viande; pourrait convenir pour préparation de viande sous vide, mais forte proportion à passer en fabrication car avants et flanchets très importants.

$\mathrm{MH}_{5}$ : convexe; couverture bonne; graisse jaune clair à jaune; belle viande, pourrait convenir au marché parisien.

$\mathrm{MH}_{2}$ : profil subconvexe à convexe; couverture correcte à légèrement insuffisante; graisse jaune clair à jaune.

$\mathrm{MH}_{4}$ : profil rectiligne à subconvexe; couver- 
ture moyenne à correcte; graisse jaune foncé; belle viande mais paraissant fatiguée (transport de Miadana); avants très importants.

$\mathrm{MH}_{6}$ : profil convexe; couverture correcte; graisse jaune clair à jaune; belle viande, belle couleur; bonne pour viande sous vide pour les arrières, proportion de gîte importante.

$\mathrm{MC}_{1}$ : profil droit à convexe; couverture correcte à légèrement insuffisante; graisse jaune clair; gras huileux; pas assez mûr pour congélation.

$\mathrm{MC}_{2}$ : profil droit à convexe; couverture correcte; graisse jaune clair.

\section{Etat de gras}

L'indice varie de 0,4 p. 100 à 2,8 p. 100 , c'est-à-dire nettement moins que pour les animaux de race Zébu Malgache. Si l'on compare le lot de métis $\mathrm{MH}_{3}$ et le lot de Zébu local $\mathrm{ZH}_{1}$, d'âges voisins et tous deux non finis pour la boucherie, le premier présente un indice de 0,4 et le second de 1,06 p. 100. De même, pour deux lots finis au concentré à volonté pendant 6 mois, le lot $\mathrm{MC}_{2}$ présente un indice de gras de 2,8 p. 100 et le lat $\mathrm{ZC}_{5}$ de 3,8 p. 100.

\section{Epaisseur de la cuisse et indice de muscle}

L'épaisseur de la cuisse varie de 22,7 à $26,7 \mathrm{~cm}$ et l'indice de 18,5 à 21,5 p. 100 . On constate que cat indice diminue avec l'âge (pour des animaux d'élevage comparable) le lot $\mathrm{MH}_{1}, 1 / 2$ Brahman d'herbe en bon état a un indice de 20,7 p. 100 et le lot $\mathrm{MH}_{\bar{v}}$, $1 / 2$ Brahman d'herbe en bon état, plus vieux de 2 ans, présente un indice de 19,5 p. 100 ; de même l'indice passe de 21,5 p. 100 à 19,4 p. 100 pour des animaux $3 / 4$ Brahman.

Il est difficile de discerner une différence entre les métis Brahman et les Zébus Malgaches; on peut dire que si cette différence existe, elle est faible, de l'ordre de 1 point. La différence est par contre nette entre les Zébus Malgaches et les métis Brahman d'une part, et les bouvillons Charollais d'autre part, traduisant les muscles relativement "plats" des premiers et les muscles "ronds " des races à viande européennes.

\section{Indice d'état de viande}

Les valeurs vont de 1,57 à 2,29 . La supério- rité des métis par rapport au Zébu Malgache est ici considérable. L'indice de gras étant nettement inférieur et l'indjce de muscle à peine supérieur, cette amélioration de l'état de viande peut s'expliquer par la conformation générale des métis qui sont plus larges et qui ont la côte plus ronde; l'indice d'état de viande supérieur chez les métis est en liaison avec la supériorité des rendements observée au chapitre précédent.

\section{Rendement en muscle}

Le rendement en muscle va de 59,1 à 64,7 p. 100 , ce qui, pour le meilleur lot, se rapproche des bouvillons Charollais qui atteignent 66,3 p. 100 . Ce bon rendement en muscle des animaux croisés jeunes, est lié à leur faible teneur en tissus gras. Les rendements des Zébus Malgaches allaient de 57,2 à 61,9 p. 100 entre les lots comparables, la différence raciale est de trois points pour le rendement en muscle.

\section{QUALITE DE LA VIANDE}

Une étude systématique de la qualité de la viande avait été effectuée pour les lots $\mathrm{ZH}_{2}$, $\mathbf{M H}_{1}$ et $\mathbf{M H}_{2}$ et publiée (15). Il s'agissait de trois lots d'animaux d'herbe, le lot de Zébus Malgaches, à croissance lente, étant bien plus vieux que les lots de croisés. Il ressortait de cette étude que la tendreté était un facteur limitant pour les zébus locaux, puisque seuls le rumsteak, l'aloyau et le filet avaient été jugés d'une tendreté satisfaisante préparés en cuisson rapide; le goût et la jutosité avaient été jugés satisfaisants. L'extension de la découpe en morceaux à cuisson rapide était bien supérieure chez les croisés.

Un des avantages de la finition du zébu local au concentré est l'amélioration de la qualité de la viande, spécialement au point de vue tendreté. La tendreté est améliorée chez les animaux jeunes finis au concentré, du fait de leur jeune âge, mais aussi chez les animaux vieux bien engraissés, la charge gnaisseuse intramusculaire améliorant fortement la qualité de la viande. Des dégustations effectuées sur les animaux du lot $\mathrm{ZC}_{5}$ (4 ans) et $\mathrm{ZC}_{9}$ (12 ans) ont confirmé cette qualité.

$\mathrm{Au}$ niveau de l'exportateur et du détaillant elle doit se traduire par une plus-value du kilogramme de viande et c'est un argument économique de plus en faveur des unités de finition. 


\section{CONCLUSION}

Cette étude a permis tout d'abord de préciser certaines données techniques.

La variation du poids du contenu digestif est très élevée (de 9 à 22 p. 100 du poids vif de départ), l'âge et surtout le mode d'alimentation étant les deux facteurs principaux de variation dans notre cas. Les normes de contenus digestifs ont pu être établies en fonction du mode d'alimentation. Au cours du transport et du jeûne avant l'abattage, la vidange, rapide au début, continue plus lentement avec le temps; l'interprétation du rendement commercial (par rapport au poids vif de départ) et surtout du rendement à jeun (par rapport au poids vif avant abattage) doit être faite avec grandes précautions si l'on ne veut pas risquer de conclusions erronnées. Le rendement vrai (par rapport au poids vif vide) est beaucoup plus fidèle.

Des normes de rendements commerciaux et de rendements vrais ont été établies, pour les races considérées et par qualité d'animal : Zébu Malgache de 40 p. 100 à 59 p. 100 , métis Brahman de 48 p. 100 à 56 p. 100 de rendement commercial (on notera que pour aucun lot de métis l'engraissement n'a été poussé à l'extrême comme c'est le cas pour les boufs de fosse). Des normes d'indice de gras, ayant pour base le poids du gras de rognon ont été établies pour le Zébu Malgache (pour les animaux jeunes où état de gras moyen correspond à un indice de 2 à 3,5 p. 100 , pour les animaux de plus de 5 ans de 1,1 à 1,6 p. 100).

Une liaison élevée semble exister pour le Zébu Malgache entre la longueur de la carcasse et l'âge de l'animal; une abaque. à étudier. senait très utile dans les abattoirs industriels.

Une liaison très forte existe entre le rendement vrai et l'indice d'état de viande; cette liaison à préciser permettrait l'étude rapide d'un grand nombre de carcasses. L'indice d'état de viande augmente avec l'âge à qualité d'état comparable.

Chez le Zébu Malgache, l'indice de gras et l'indice de muscle utilisés dans cette étude varient dans le même sens sauf exception, ce qui contrarie les prévisions de rendement musculaire de la carcasse.

Le pourcentage de gras et le rendement en muscle qui lui est lié semblent être les carac- tères raciaux, les métis Brahman présentant un rendement musculaire de trois points plus élevés que les Zébus Malgaches comparables par ailleurs; comme il s'agit d'animaux croisés, l'hétérosis entre certainement pour une part dans cette amélioration.

Dans l'optique de l'étude et de l'amélioration de la production de viande à Madagascar, on peut dégager les idées suivantes :

Les Zébus Malgaches, s'ils ne sont pas en bon état, donnent des rendements très mauvais (rendement commercial 40 à 45 p. 100). Pour présenter une certaine valeur, ils doivent impérativement être en bon état. Pour qu'ils soient en état à l'herbe il faut qu'ils soient d'un âge assez avancé et qu'ils disposent d'un pâturage relativement bon; ces conditions ne sont remplies que pour une partie du bétail, en particulier à cause de "l'aspiration" du marché qui demande un nombre d'animaux trop élevé, et presque constant au cours de l'année, ce qui nécessite souvent la commercialisation d'animaux trop jeunes ou trop maigres. La qualité des carcasses varie largement au cours de l'année.

L'embouche paysannale, permet de remédier en partie à cet inconvénient en faisant passer des animaux insuffisants pour animaux en bon état, pouvant atteindre le niveau des meilleurs Zébus Malgaches d'herbe (58-62 p. 100 de rendement vrai).

Cette embouche paysannale, bien qu'actuellement encouragée, n'est pas extensible à tous les animaux pour des raisons complexes (techniques, économiques, sociales). Une finition industrielle permet d'atteindre, avec des animaux plus jeunes, des rendements satisfaisants (58 p. 100 de rendement commercial). Elle a l'avantage de libérer les pâturages extensifs des zones naisseuses permettant, théoriquement, d'augmenter le cheptel de reproducteurs; elle permet de pousser pratiquement la totalité des animaux engraissés au niveau des meilleurs Zébus; elle permet la commercialisation de viande plus jeune, mieux appréciée sur le marché de l'exportation; enfin, argument important, elle permet d'étaler les abattages sur toute l'année ce qui est impossible en n'utilisant que des animaux d'élevage extensif. Il faut noter que le Zébu Malgache a tendance à s'engraisser exagérément, ce qui entraîne un rendement en muscle relativement peu élevé; il est possible 
d'obtenir de très bons rendements en carcasses mais uniquement au prix d'un excès de graisse. De même, la race étant de petit format $(400 \mathrm{~kg}$ de poids adulte pour les mâles) et présentant une croissance lente, l'obtention de carcasses de $200 \mathrm{~kg}$ par finition intensive d'animaux trop jeunes (3-5 ans) donne des carcasses trop grasses. Il semble préférable de finir au concentré des animaux de 6 à 8 ans pour obtenir des carcasses de $200 \mathrm{~kg}$ et de se contenter de carcasses plus légères $(180 \mathrm{~kg}$ ) lorsque l'on doit finir des animaux de 3 à 5 ans. La spéculation traditionnelle du bcuf de fosse subissant une finition très poussée permet d'abtenir des animaux très gras (66 p. 100 de rendement vrai), réservés à une clientèle aux goûts particuliers.
L'améliosation génétique seule apporte un progrès considérable qui se traduit par des carcasses plus lourdes d'animaux jeunes, des rendements en carcasses et des rendements en muscles supérieurs; cette solution est employée chez les éleveurs privés de plusieurs régions de Madagascar et dans les ranchs d'Etat.

La finition avec une alimentation concentrée d'animaux de races améliorées permet des croissances plus rapides $(200 \mathrm{~kg}$ de carcasses à 16 - 18 mois), un meilleur indice de consommation, des rendements en carcasses et des rendements musculaires comparables à ceux des animaux croisés élevés à l'herbe, et l'obtention d'une viande très tendre due au jeune âge.

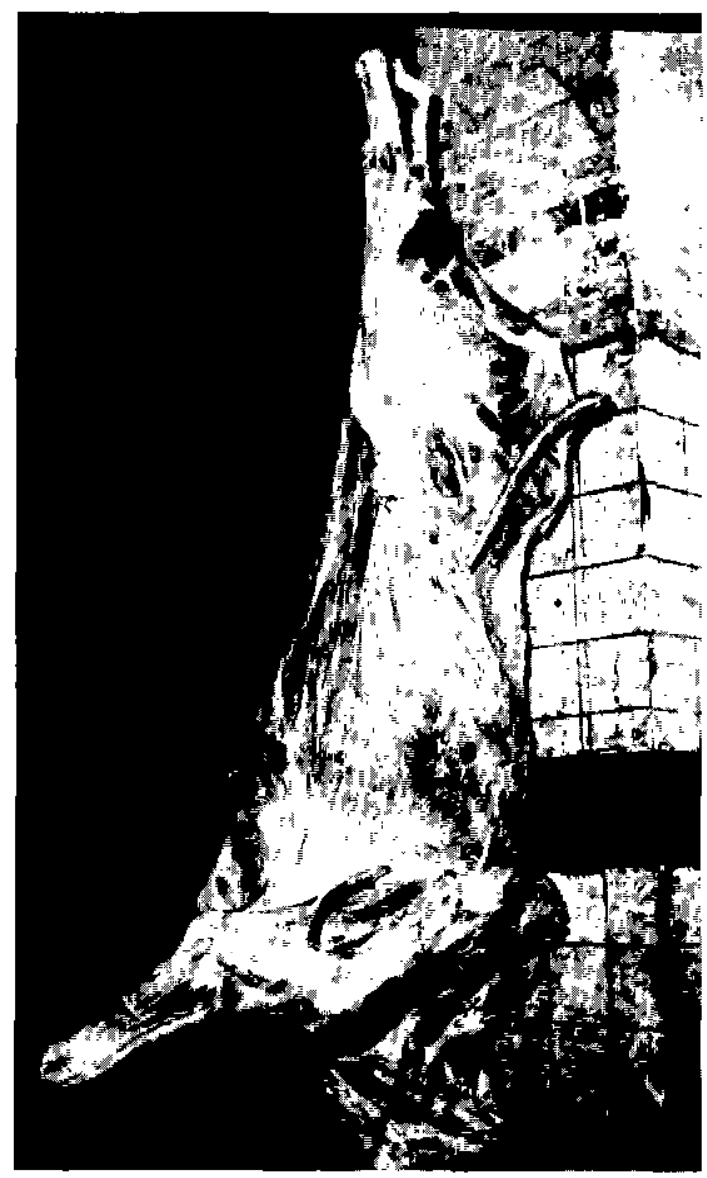

Photo ñ '1. - Lot ZH 1. Zébu malgache d'herbe maigre. Age : 4 ans 9 mois. Carcasse: $144 \mathrm{~kg}$.

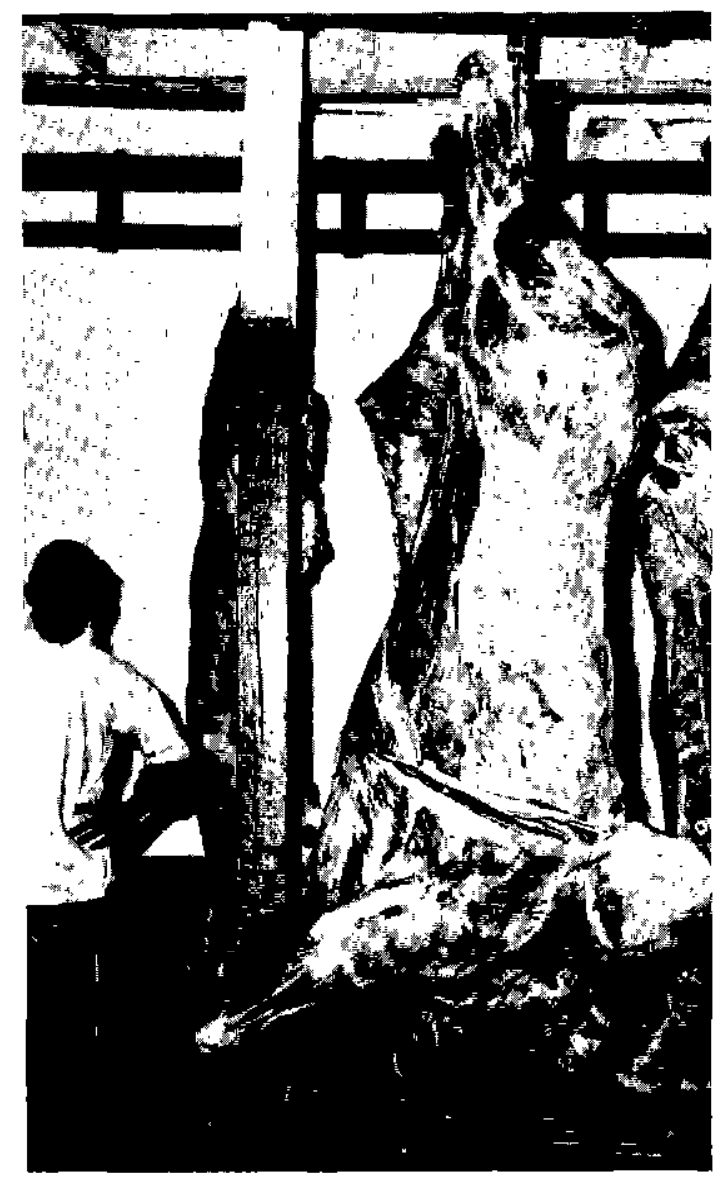

Photo no 2. - Lot ZC 3. Zébu malgache engraissé. Age : 4 ans. Carcasse : $180 \mathrm{~kg}$. 


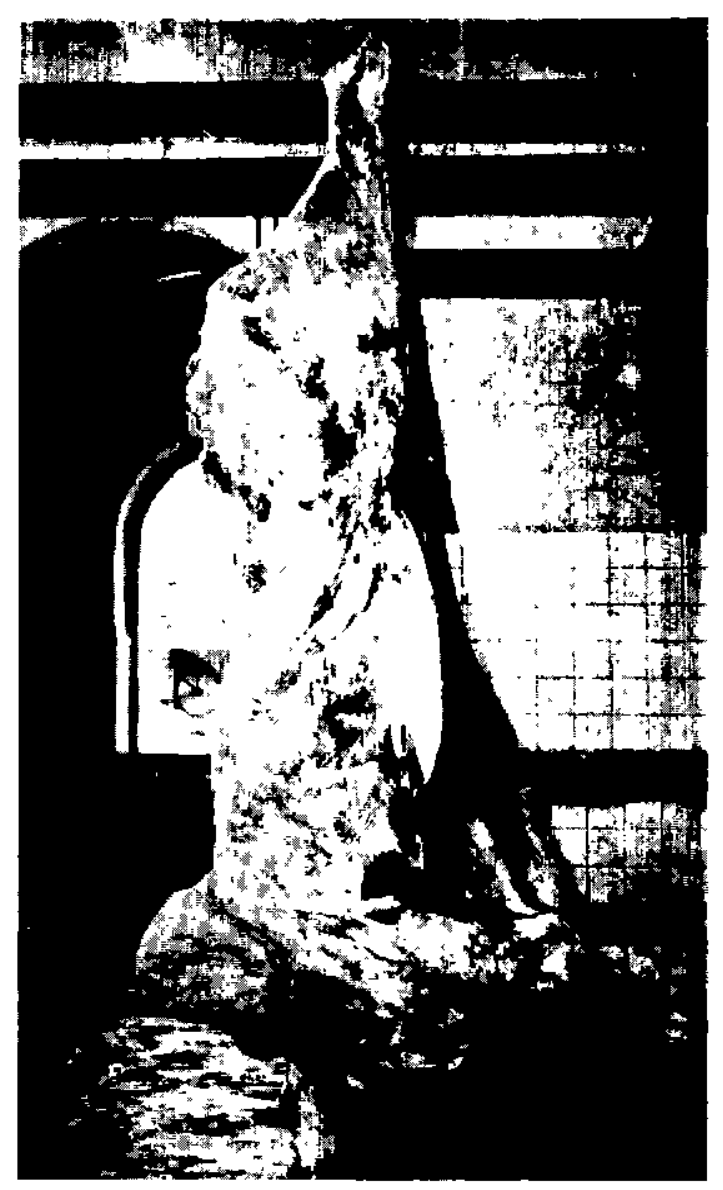

Photo no 3. - Lot ZC 8. Zébu malgache engraissé. Age: 9 ans 3 mois. Carcasse: $216 \mathrm{~kg}$.

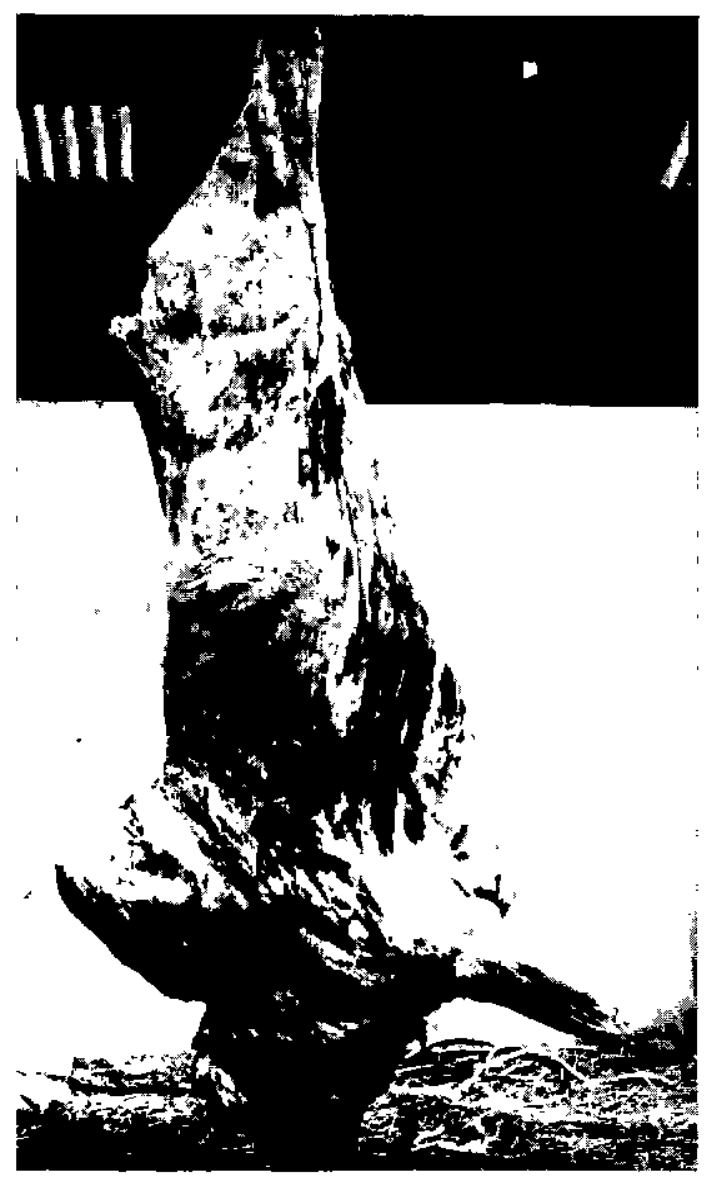

Photo $n^{\circ}$ 4. - Lot ZC 9. Zébu malgache (bouf de fosse). Age: 12 ans. Carcasse : $274 \mathrm{~kg}$. 


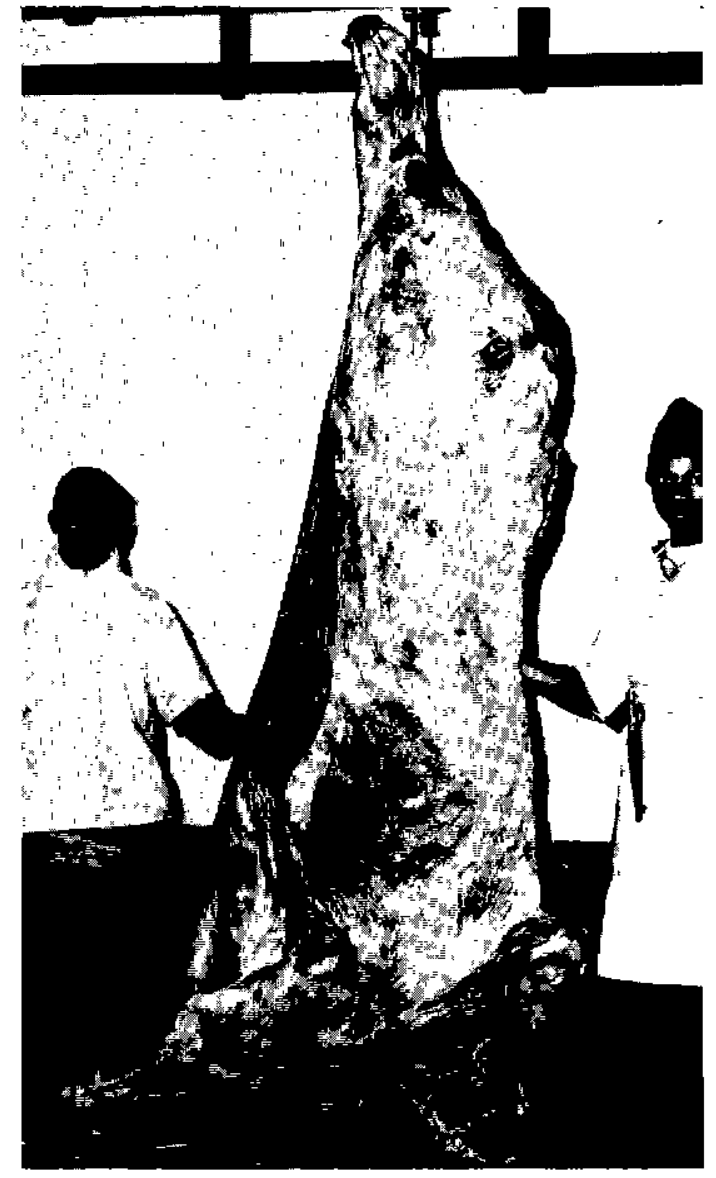

Photo no 5. - Lot MH 5. Métis Brahman X Zébu malgache. Age: 5 ans 6 mois. Carcasse : $314 \mathrm{~kg}$.

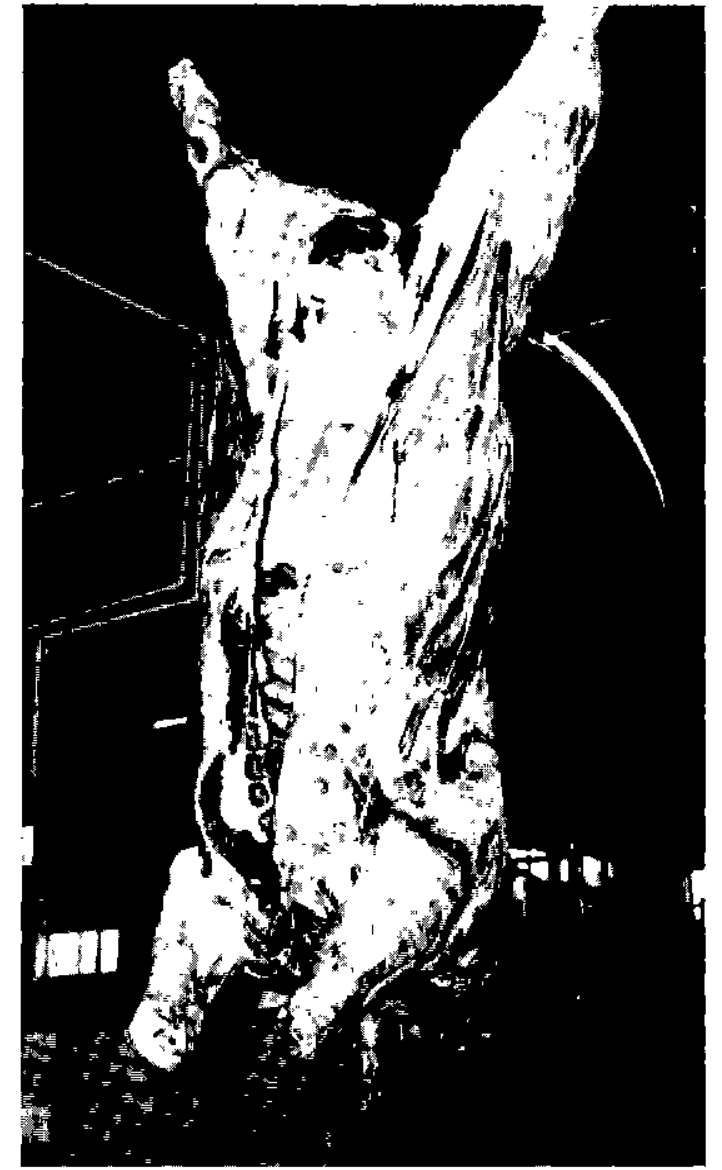

Photo $n^{0}$ 6. - Lot MC 1. Métis Frison $\times$ Zébu. Age : 18 mois. Carcasse : $219 \mathrm{~kg}$.

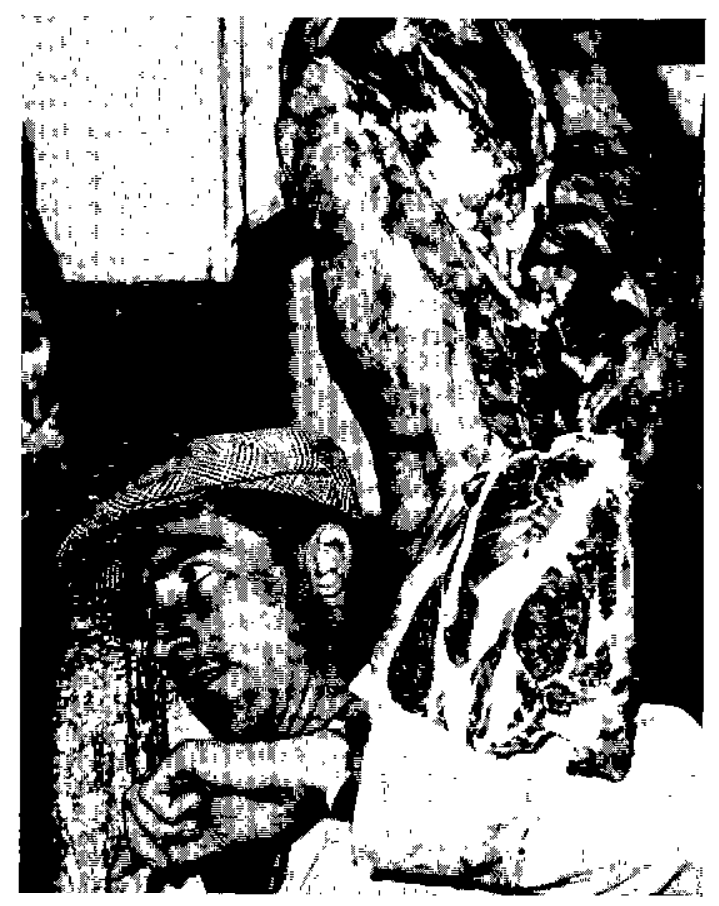

Photo $\mathbf{a}^{0}$ 7. - Lot ZC 5. Zébu malgache engraissé. Rapport muscle/graisse $=$ faible. 


\section{SUMMARY}

\section{Meat production characteristics of zebu cattle in Madagascar}

The Author studies comparatively the meat production characteristics of Malagasy Zebu cattle reared in natural pasture, completed natural pasture, feed-lot an in "pits". After, he studies in conditions of completed or not natural pasture, then in feed-lot, Brahman Zebu $\times$ Malagasy Zebu cross-bred. A lot contanns Malagasy Zebu $X$ Friesland (or Norman) cattle. quality.

The intensive feed and the crossings lead to an improvment of carcass

\section{RESUMEN}

\section{Valor carnicero de cebues en Madagascar}

El autor compara los valores carniceros de cebues malgachos criados a pasto natural, a pasto natural con aditivos, en feed-lot, $y$ en « hoyo». Luego estudia en las condiciones de pasto natural con o sin aditivos, y en feed-lot animales mestizos cebú Brahman $\times$ cebú malgacho. Mestizos cebú malgacho $\times$ Frison (o Normand) constituyen un lote. La alimentación intensiva y los cruzamientos dan una mejora de la cualidad de las canales.

\section{BIBLIOGRAPHIE}

1. CHAUdieU (G.), "Pour le Boucher", Paris, Dunod, 1959.

2. CHAUdieU (G.), BONNEVille (A.), "Boucher de Paris ", tome I: "Le bouf ", Paris, Peyronnet, 1950.

3. CHRETIEN (M.), "Le bouf de Madagascar envisagé comme animal de boucherie", Rev. Zootech. 1922 (5) : 1-17.

4. CRAPLET (C.), "La viande de bovins ", 3 vol., Paris, Vigot frères, 1966.

5. DUMONT (B.L.), «Critère de l'appréciation de la production de viande », Rame. Fédération Européenne de Zootechnie, 1963.

6. DUMONT (B. L.) et collab., «Observations sur les qualités de boucherie des taurillons Charollais », Bull. techn. I.N R.A., 1963 (1).

7. DUMONT (B. L.), LE GUELTE (P.). ARNOUX (J.), « Etude biométrique des bovins de boucherie. 1. Variabilité de la composition anatomique de la carcasse des bovins charollais ", Ann. Zoot. 1961, 10 (3) : 149-54; \& 2 Estimation des poids de la musculature chez les bovins charollais », Ann. Zoot. 1961, 10 (4): 321-26; * 3 Variabilité de la composition en morceaux et des caractères de la composition de la carcasse des boufs charollais ", Ann. Zoot. 1964, 13 (3) : 255-66.

8. LACROUTS (M.) et collab., « Etude des problèmes posés par l'élevage et la commercialisa- tion du bétail et de la viande à Madagascar», 2 vol., Paris, Ministère de la Coopération, 1962.

9. LE GUELTE (P.), « Comparaison des caractéristiques de conformation et composition des carcasses de trois types de jeunes bovins charollais \#, Bull. techn. I.N.R.A., 1964 (4).

10. METZGER (G.), HAMON (J. L.), « Engraissement précoce et abattage expérimental de trois lots de bovins: zébu, demi-sang Brahman et Afrikander-Limousin-Zébu ", Bull. Madagascar 1959 (157) : 471-81.

11. RENOU (Y.), « Note sur les résultats des épreuves de consommation organisées à l'occasion du concours général agricole $1963 \%$, Bull. techn. I.N.R.A. 1964 (3).

12. REVIERS (B., de), GILIBERT (J.), SERRES (H.), «Aljmentation intensive des zébus à Madagascar », Région de Madagascar, Note de l'I.E.M.V.T., 19 p.

13. SARNIGUET (J.) et collab., " L'embouche bovine sur les hauts plateaux malgaches», Paris, Secrétariat d'Etat aux Affaires étrangères, 1969.

14. SERRES (H.), "L'engraissement des zébus dans la région de Tananarive selon la technique du bouf de fosse", Rev. Elev. Méd vét. Pays trop. 1969,22 (4) : $529-38$

15. SERRES (H.) et collab., *Le croisement Brabman à Madagascar », Rev. Elev. Méd. vét. Pays trop., 1968, 21 (4) : 519-61. 